\title{
Longitudinal Association Between Oral Status and Depressive Symptoms Among Chinese Older Adults - China, 2014-2018
}

\author{
Xinhui Zhang'; Xiyuan $\mathrm{Hu}^{1}$; Yalu Zhang ${ }^{1}$; Jingjing Sun ${ }^{1}$; Gong Chen $^{1, *}$
}

\section{Summary \\ What is already known about this topic?}

Previous studies on the importance of oral health in later life have mainly focused on nutritional and physical health indicators, while the effects of oral status on the mental health of older people, especially on depression, need to be further explored.

What is added by this report?

Longitudinal results show tooth loss was positively associated with depressive symptoms among older adults in the mainland of China, while denture use was associated with a decreased risk of depression. Effect modification by denture use was observed ( $P$ for interaction <0.001).

What are the implications for public health practice?

Measures should be taken to help Chinese older adults promote oral health and strengthen the care of dentures by expanding basic health insurance coverage to include dental prosthodontics or providing affordable dental insurance for seniors.

Oral status, primarily marked by tooth loss, is critical for maintaining physical, mental, and social wellbeing (1), especially in later life. Prevalence and incidence estimates of severe tooth loss increased gradually with age (2). Meanwhile, late-life depression is a significant public health problem, which can often result in personal suffering, family disruption, and the deterioration of many medical disorders (3). The relationship between oral status and depressive symptoms among older adults, while receiving increasing amounts of attention, has not yet been studied conclusively. Some research based on American adults found that tooth loss was associated with depression (4). However, some studies reported that poor dental health was not significantly associated with depressive symptoms among the elderly (5). The evidence about the relation between oral status and late-life depression is still limited and inconsistent. Additionally, although there are a few cross-sectional results focusing on Chinese older adults ( $)$, more longitudinal studies are still needed to target the association between oral status, including tooth loss and denture use, and depressive symptoms among Chinese older adults.

Therefore, this study aims to explore the relationship between oral status, including tooth loss and denture use, and depressive symptoms among older Chinese adults using data from the Chinese Longitudinal Healthy Longevity Survey (CLHLS). Furthermore, the combined association of tooth loss and denture use with depressive symptoms was explored as well as interaction effects by different demographic and behavioural characteristics.

The data used in this paper came from CLHLS. Focusing on the older population aged 65 and above, the CLHLS was conducted in 1998-2018 from randomly selected counties and cities of 22 out of 31 provincial-level administrative divisions (PLADs) in China (7). We used 2 waves of CLHLS data conducted in 2014 and 2018 to analyze how tooth loss and denture use in 2014 influenced depressive symptoms 4 years later (8). Overall, 3,310 out of 7,192 eligible participants were included for analyses after excluding cases lost to follow-up and with missing information on key variables (see Supplementary Figure S1 for the variable screening process, available in http:// weekly.chinacdc.cn/). We collected the self-reported number of natural teeth and classified the participants into 4 categories (0, $1-9,10-19$, and 20 and above). A five-item scale, which has been applied in several studies to represent depressive symptoms via the CLHLS data (9), was adopted in the CLHLS to evaluate depressive symptoms with higher values indicating more depressive symptoms. The definitions of other variables are shown in the Supplementary Materials (available in http://weekly.chinacdc.cn/).

Supplementary Table S1 (available in http://weekly. chinacdc.cn/) shows baseline characteristics of elderly by numbers of natural teeth and denture use. The mean age of the participants was $81.29(\mathrm{SD}=10.32)$, $46.99 \%$ were male, $39.13 \%$ had an urban residence, 
$71.60 \%$ of the participants had 19 teeth or less, and $28.40 \%$ had completely no teeth. The denture use rate was $39.40 \%$. The mean of depressive symptom scores was $6.78(\mathrm{SD}=3.19)$ and increased with tooth loss. Participants using dentures had lower depressive symptom scores compared with those without the denture. Supplementary Table S2 (available in http:// weekly.chinacdc.cn/) further demonstrate depressive Symptom Scores of the participants in 2018 by Covariates.

This study followed a stepwise approach and applied multivariable linear models to adjust for confounding variables to clarify the effects of oral status on depressive symptoms. Model 1 only included categories of natural teeth numbers and denture use. Model 2 adjusted for the demographic and socioeconomic covariates, such as age, gender, ethnicity, marital status, residence, education, and pension. Model 3 further added the covariates of lifestyle, including smoking, alcohol consumption, fruit intake, vegetable intake, and health conditions including Activities of Daily Living (ADL) and MiniMental State Examination (MMSE) score. Model 4 further controlled for variables of social engagement, including playing cards or mahjong, participation in community activities, and travel. Model 4 was identified as a fully adjusted model. Subgroup analysis was conducted to assess differences between groups. The mediation effects were tested by adding interaction terms of the number of natural teeth and age (65-79 years or $\geq 80$ years), gender, ethnicity, smoking, drinking, and denture use. All statistical analyses were performed using the STATA 16 software (MP version16.0, StataCorp LLC, USA). $P<0.05$ was considered to be statistically significant.

Table 1 presented the regression results of the associations of the numbers of natural teeth and status of denture use with depressive symptoms. The depressive symptom scores increased and slightly decreased as the numbers of teeth decreased ( $P$ for trend <0.001). For the fully adjusted model, individuals with $10-19,1-9$, and 0 teeth had 0.55 , 0.59 , and 0.33 point higher scores of depressive symptoms than those with $20+$ teeth, respectively (all $P<0.01)$. The denture users had an adjusted lower score of 0.27 points $(P<0.01)$ compared with people without dentures.

As the association of the interaction of tooth loss and denture use with depressive symptoms was significant (Table 2; $P$ for interaction $<0.001$ ), we showed the combined effects of tooth loss and denture use on depressive symptoms in Table 3. Among nondenture users, scores of depressive symptoms compared with those who had 20+ teeth with/without dentures were 0.64 and 0.61 points higher for older adults with 10-19 teeth and $1-9$ teeth, respectively (all $P<0.001$ ). Denture users had a lower score than non-users in the category of 1-9 teeth. However, there was no significant difference between denture/non-denture users with 20 teeth and denture users with 10-19 teeth, as well as non-denture users with 0 teeth. It is worth noting that denture users with 0 teeth as compared with those with $20+$ teeth still had a significantly higher score of depressive symptoms

TABLE 1. Association between number of natural teeth, denture use, and depressive symptom scores among Chinese older adults - China, 2014-2018.

\begin{tabular}{|c|c|c|c|c|}
\hline \multirow{2}{*}{ Item } & \multicolumn{4}{|c|}{ Coefficient $(95 \% \mathrm{Cl})$ for depressive symptom $(\mathrm{N}=3,310)$} \\
\hline & Model 1 & Model 2 & Model 3 & Model 4 \\
\hline \multicolumn{5}{|c|}{ Number of natural teeth } \\
\hline $20+$ & Reference & Reference & Reference & Reference \\
\hline $10-19$ & $0.95(0.90,1.25)^{\star \star \star}$ & $0.62(0.35,0.71)^{\star \star \star}$ & $0.57(0.34,0.70)^{\star \star \star}$ & $0.55(0.32,0.68)^{\star \star \star}$ \\
\hline $1-9$ & $1.21(1.29,1.60)^{\star * *}$ & $0.69(0.42,0.77)^{\star * *}$ & $0.60(0.32,0.67)^{\star * *}$ & $0.59(0.30,0.65)^{\star * *}$ \\
\hline 0 & $1.15(1.25,1.57)^{\star * *}$ & $0.47(0.21,0.59)^{\star * *}$ & $0.37(0.06,0.44)^{* *}$ & $0.33(0.03,0.41)^{\star \star}$ \\
\hline$P$ for trend & $<0.001$ & $<0.001$ & $<0.001$ & $<0.001$ \\
\hline \multicolumn{5}{|l|}{ Denture use } \\
\hline No & Reference & Reference & Reference & Reference \\
\hline Yes & $-0.83(-0.96,-0.71)^{\star * *}$ & $-0.40(-0.50,-0.24)^{\star \star *}$ & $-0.24(-0.36,-0.09)^{\star * *}$ & $-0.27(-0.32,-0.06)^{\star \star}$ \\
\hline
\end{tabular}


(coefficient $=0.20, P<0.05)$.

No significant interaction effects were found in subgroup analyses for the number of natural teeth by ethnicity, smoking, and drinking (Table 3). Of note is that older adults with 1-9 teeth were associated with a higher depressive symptom score in the older elderly of

TABLE 2. The combined effects of tooth loss and denture use on depressive symptom scores among Chinese older adults - China, 2014-2018.

\begin{tabular}{|c|c|c|c|c|}
\hline \multirow{2}{*}{ Oral status } & \multicolumn{4}{|c|}{ Coefficient $(95 \% \mathrm{Cl})$ for depressive symptom $(\mathrm{N}=3,310)$} \\
\hline & Model 1 & Model 2 & Model 3 & Model 4 \\
\hline $20+$ teeth & Reference & Reference & Reference & Reference \\
\hline $10-19$ teeth with dentures & $0.15(-0.22,0.53)$ & $0.17(-0.12,0.71)$ & $0.15(-0.12,0.69)$ & $0.18(-0.12,0.69)$ \\
\hline 10-19 teeth without dentures & $1.10(0 \cdot 99,1 \cdot 38)^{\star * *}$ & $0.71(0.40,0.79)^{* * *}$ & $0.66(0.39,0.79)^{* * *}$ & $0.64(0.40,0.87)^{* * *}$ \\
\hline 1-9 teeth with dentures & $0.42(0.12,0.73)^{\star *}$ & $0.32(0.02,0.62)^{*}$ & $0.35(0.26,0.98)^{\star *}$ & $0.37(0.23,0.96)^{*}$ \\
\hline 1-9 teeth without dentures & $1.37(1.15,1.59)^{* * *}$ & $0.76(0.52,0.99)^{* * *}$ & $0.64(0.40,0.87)^{\star * *}$ & $0.61(0.38,0.85)^{* * *}$ \\
\hline 0 teeth with dentures & $0.49(0.25,0.72)^{\star \star *}$ & $0.18(-0.06,0.42)$ & $0.22(0.11,0.79)^{*}$ & $0.20(0.04,0.45)^{*}$ \\
\hline 0 teeth without dentures & $1.21(0.93,1.48)^{\star * *}$ & $0.43(0.13,0.56)^{\star \star}$ & $0.25(-0.11,0.33)$ & $0.23(-0.13,0.31)$ \\
\hline
\end{tabular}

Abbreviation: $\mathrm{Cl}=$ confidence interval.

${ }^{*} P \leq 0.05$.

${ }^{* *} P \leq 0.01$

${ }^{* * *} P \leq 0.001$.

TABLE 3. Subgroup analysis of the association between number of natural teeth and depressive symptom scores among Chinese older adults - China, 2014-2018.

\begin{tabular}{|c|c|c|c|c|c|}
\hline \multirow{2}{*}{ Subgroup } & \multicolumn{4}{|c|}{ Number of natural teeth $(95 \% \mathrm{CI})$} & \multirow{2}{*}{$P$ for interaction } \\
\hline & 0 & $1-9$ & $10-19$ & $20+$ & \\
\hline Age & & & & & $<0.001$ \\
\hline $65-79$ years & $0.48(0.17,0.79)^{\star *}$ & $0.31(0.05,0.58)^{*}$ & $0.55(0.32,0.78)^{\star \star \star}$ & Reference & \\
\hline$\geq 80$ years & $0.16(-0.11,0.44)$ & $0.54(0.28,0.80)^{\star * \star}$ & $0.49(0.20,0.77)^{\star \star}$ & Reference & \\
\hline Gender & & & & & 0.021 \\
\hline Women & $0.14(-0.14,0.42)$ & $0.42(0.15,0.68)^{\star \star *}$ & $0.53(0.26,0.80)^{\star \star \star}$ & Reference & \\
\hline Men & $0.29(0.02,0.55)^{*}$ & $0.51(0.28,0.75)^{\star * \star}$ & $0.42(0.18,0.66)^{\star \star \star}$ & Reference & \\
\hline Ethnicity & & & & & 0.548 \\
\hline Han & $0.28(0.01,0.41)^{*}$ & $0.58(0.30,0.67)^{\star \star *}$ & $0.57(0.29,0.66)^{\star \star \star}$ & Reference & \\
\hline Minorities & $0.59(-0.16,1.19)$ & $0.62(-0.21-0.95)^{*}$ & $0.97(0.12,1.29)^{\star \star}$ & Reference & \\
\hline Smoking & & & & & 0.107 \\
\hline Never smoke & $0.19(-0.21,0.58)$ & $0.78(0.34,1.21)^{\star * \star}$ & $0.47(0.24,0.70)^{\star \star \star}$ & Reference & \\
\hline All the time & $0.18(-0.21,056)$ & $0.78(0.35,1.21)^{\star * *}$ & $0.48(0.26,0.71)^{\star * *}$ & Reference & \\
\hline Stop smoking & $0.35(-0.06,0.76)$ & $0.36(-0.11,0.83)$ & $0.18(-0.07,0.43)$ & Reference & \\
\hline Drinking & & & & & 0.309 \\
\hline Never drink & $0.39(-0.02,0.80)$ & $0.90(0.42,1.39)^{\star *}$ & $0.44(0.22,0.67)^{\star * *}$ & Reference & \\
\hline All the time & $0.19(-0.22,0.60)$ & $0.64(0.18,1.10)^{\star \star}$ & $0.53(0.31,0.75)^{\star \star \star}$ & Reference & \\
\hline Stop drinking & $0.29(-0.16,0.75)$ & $0.24(-0.29,0.76)$ & $0.23(-0.01,0.47)^{*}$ & Reference & \\
\hline Denture use & & & & & $<0.001$ \\
\hline Yes & $0.60(-0.15,0.65)^{\star *}$ & $0.71(0.21,0.95)$ & $0.48(0.07,0.77)$ & Reference & \\
\hline No & $0.11(0.35,0.75)^{\star \star \star}$ & $0.51(0.24,0.65)^{\star * *}$ & $0.56(-0.15,0.33)^{*}$ & Reference & \\
\hline
\end{tabular}

Note: Coefficients were based on linear regression model 4. Effect modification by age ( $P$ for interaction $<0.001)$, gender $(P$ for interaction $=0.021)$, and denture use $(P$ for interaction $<0.001)$.

Abbreviation: $\mathrm{Cl}=$ confidence interval.

${ }^{*} P \leq 0.05$.

** $P \leq 0.01$.

$* * * P \leq 0.001$. 
$80+($ coefficient $=0.54, P<0.001)$ than in the younger elderly of 65-79 years (coefficient $=0.31, P<0.05$ ), while there existed inverse relationship among people with no teeth and those with 10-19 teeth ( $P$ for interaction <0.001). In addition, the depressive symptom scores of men were higher than those of women with regard to people with 9 teeth or less, and the scores of women were higher than those of men for participants with $10-19$ teeth $(P$ for interaction $=0.021$ ).

\section{DISCUSSION}

The primary findings of this prospective study were that there was a significant association between oral status and depressive symptoms of Chinese older adults at 4-year follow-up, which meant that participants with fewer teeth left $(<20)$ and those who were nondenture users were associated with severer depressive symptoms. We also found that the association between tooth loss and depressive symptoms seemed to be mitigated by wearing dentures in each tooth loss category, considering the combined effects of tooth loss and denture use on depressive symptom scores. Subgroup analysis showed that young older adults (65-79 years) and men with tooth loss were more likely to suffer from depressive symptoms.

Our findings were consistent with prior studies (9-12). A prospective longitudinal study found that older adults in Brazil who experienced tooth loss were at greater risk of exhibiting depressive symptoms (9). In a Japanese longitudinal study, having no teeth could play a role in worsening depressive symptoms in individuals aged 65 years and older (10). Another study pointed out that a deterioration in oral health increased the risk of depressive symptoms among a group of older adults in England (11). In addition, a cross-sectional study of the Republic of Korea reported that the use of dental prosthesis in patients with the loss of multiple teeth was expected to reduce the likelihood of severe depression (12). Nevertheless, a limited number of studies have investigated the independent and combined effects of both tooth loss and denture use on later life depression using largescale longitudinal data.

Several mechanisms could explain the association between oral health, referring to the number of natural teeth and denture use, and depressive symptoms. First, oral status can affect dietary intake and nutritional status (13). Several studies have suggested that diet and nutrition play an important role in preventing and managing depression (14). Denture use can contribute to the decrease of depressive symptoms by helping people keep good nutritional condition. Second, oral health is not only related to the ability of speaking, smiling, smelling, tasting, touching, chewing, and swallowing but also undertaking social functions such as expressing multiple feelings (15). Older adults may feel less confident and unnatural because of the changes in their face and way of speaking caused by tooth loss. Denture use can relieve their mental stress and depression by maintaining their previous status. Third, poor dental health predicted becoming homebound among older adults ( $\sigma$ ), and the elderly's social engagement is thus restricted. Social involvement is especially associated with depressive symptoms among the elderly.

Oral health, effectively marked by tooth loss, is of great significance to the psychological wellbeing of older adults. However, Chinese older adults who suffer tooth loss do not pay enough attention to oral health due to the financial burden of dental care and poor oral health literature. It is urgent to take measures to help senior citizens promote oral health and strengthen the care of dentures by expanding basic health insurance coverage to include dental prosthodontics or by providing affordable dental insurance for seniors.

The study was subject to some limitations. First, the relationship between oral status and depressive symptoms could only be concluded as an association rather than causation because the study design was observational. Second, misclassification bias could arise from the loss of detailed information, such as time of tooth loss and type of denture use. Third, residual confounding may exist as a result of the presence of unmeasured or unknown factors. Fourth, the quality of adopted 5-item scale of depressive symptoms might have been worse than other depression scales such as the Geriatric Depression Scale (GDS) or Center for Epidemiologyical Studies Depression Scale (CES-D). Last, self-reported information of numbers of teeth may lead to imprecise measurements.

Acknowledgements: All CLHLS participants and relevant staff.

Conflicts of interest: No conflicts of interest.

Funding: The National Social Science Foundation (N20ZDA076).

doi: $10.46234 / \mathrm{ccdcw} 2021.208$

\#Corresponding author: Gong Chen, chengong@pku.edu.cn.

${ }^{1}$ Institute of Population Research, Peking University, Beijing, China.

Submitted: September 14, 2021; Accepted: September 28, 2021 


\section{REFERENCES}

1. Sischo L, Broder HL. Oral health-related quality of life: what, why, how, and future implications. J Dent Res 2011;90(11):1264-70. http://dx.doi.org/10.1177/0022034511399918.

2. Kassebaum NJ, Bernabé E, Dahiya M, Bhandari B, Murray CJL, Marcenes W. Global Burden of severe tooth loss: a systematic review and meta-analysis. J Dent Res 2014;93(S7):20S - 8S. http://dx.doi.org/ $10.1177 / 0022034514537828$.

3. Alexopoulos GS. Depression in the elderly. Lancet 2005;365 (9475):1961 - 70. http://dx.doi.org/10.1016/s0140-6736(05)66665-2.

4. Matsuyama Y, Jürges H, Dewey M, Listl S. Causal effect of tooth loss on depression: evidence from a population-wide natural experiment in the USA. Epidemiol Psychiatr Sci 2021;30:e38. http://dx.doi.org/ $10.1017 /$ s2045796021000287.

5. Koyama S, Aida J, Kondo K, Yamamoto T, Saito M, Ohtsuka R, et al. Does poor dental health predict becoming homebound among older Japanese? BMC Oral Health 2016;16(1): 51. http://dx. doi.org/10.1186/s12903-016-0209-9.

6. Woo J, Ho S, Lau J, Yuen YK. Chewing difficulties and nutritional status in the elderly. Nutr Res 1994;14(11):1649 - 54. http://dx.doi.org/ 10.1016/S0271-5317(05)80319-7.

7. Zeng Y. Chinese longitudinal healthy longevity survey and some research findings. Geriatr Gerontol Int 2004;4(S1):S49 - 52. http://dx. doi.org/10.1111/j.1447-0594.2004.00147.x.

8. Kunrath I, Ribeiro Silva AE. Oral health and depressive symptoms among older adults: longitudinal study. Aging Ment Health 2020. http://dx. doi.org/10.1080/13607863.2020.1855104.

9. Shen K, Zhang B, Feng QS. Association between tea consumption and depressive symptom among Chinese older adults. BMC Geriatr 2019;19(1):246. http://dx.doi.org/10.1186/s12877-019-1259-z.

10. Yamamoto T, Aida J, Kondo K, Fuchida S, Tani Y, Saito M, et al. Oral health and incident depressive symptoms: JAGES project longitudinal study in older Japanese. J Am Geriatr Soc 2017;65(5):1079 - 84 . http://dx.doi.org/10.1111/jgs.14777.

11. Rouxel P, Tsakos G, Chandola T, Watt RG. Oral health-a neglected aspect of subjective well-being in later life. J Gerontol Ser B Psychol Sci Soc Sci 2018;73(3):382 - 6. http://dx.doi.org/10.1093/geronb/gbw024.

12. Shim J, Lee Y, Hong SJ, Noh K, Pae A, Kim HS, et al. Relationship between usage of removable denture and depression in Korean adults with loss of multiple teeth: A cross-sectional study using the seventh Korea national health and nutrition examination survey. J Korean Acad Prosthodont 2021;59(1):1 - 10. http://dx.doi.org/10.4047/jkap.2021. 59.1.1.

13. Nowjack-Raymer RE, Sheiham A. Numbers of natural teeth, diet, and nutritional status in US adults. J Dent Res 2007;86(12):1171-5. http://dx.doi.org/10.1177/154405910708601206.

14. Upton N. Developing our understanding of nutrition in depression. $\mathrm{Br}$ J Nutr 2021. http://dx. doi.org/10.1017/S0007114521001744.

15. Glick M, Williams DM, Kleinman DV, Vujicic M, Watt RG, Weyant RJ. A new definition for oral health developed by the FDI World Dental Federation opens the door to a universal definition of oral health. Br Dent J 2016;221(12):792 - 3. http://dx.doi.org/10.1038/sj. bdj.2016.953. 


\section{SUPPLEMENTARY MATERIALS}

\section{Supplementary Information of Sample Selection and Variables}

Self-reported number of natural teeth and the use of dentures were collected by use of the questions of "A: how many natural teeth (teeth that are naturally grown) do you still have?" and "B: do you have false teeth? (false teeth referred to any type of non-natural teeth, including partial or complete, removable, or implant-retained fixed dentures)." The remaining teeth were classified into four categories: $(0,1-9,10-19$, and 20 plus). We assessed the combined effects by sorting the participants into eight categories based on teeth number and denture use.

A five-item scale, which has been applied in several studies to represent depressive symptoms via the Chinese Longitudinal Healthy Longevity Survey (CLHLS) data (1-2), was adopted in CLHLS to evaluate depressive symptoms. Two questions, including "do you look on the bright side of things?" and "are you as happy now as when you were young?" measured positive feelings while three questions measured negative feelings, including the following: "do you often feel anxious or fearful ?" "do you often feel lonely and isolated?" and "do you feel the older you get, the more useless you are?". The interviewees answered those questions by choosing from five frequency options of "Always," "Often," "Sometimes," "Seldom," and "Never." Each response was assigned a value from 0 to 4 , and a higher frequency of negative feeling indicated a higher value. Then the summed value varied from 0 to 20 . Cronbach a coefficient assessed the internal consistency reliability based on the 2014 sample is 0.84 , which is higher than the acceptable values of 0.6. We operated principal component analysis to generate one factor explaining $93 \%$ of the total variance with eigenvalues $\geq 1$.

We controlled for three groups of covariates that could be significantly associated with depression of older adults according to previous studies (3-5). The first group consisted of the demographic and socioeconomic factors, including age, gender (male or female), ethnicity (Han or minority groups), marital status (married or non-married), residence (urban or rural), years of education, and pension status (having a pension or not). The second group included lifestyle and health characteristics, including smoking (current smoker, former smoker or non-smoker),

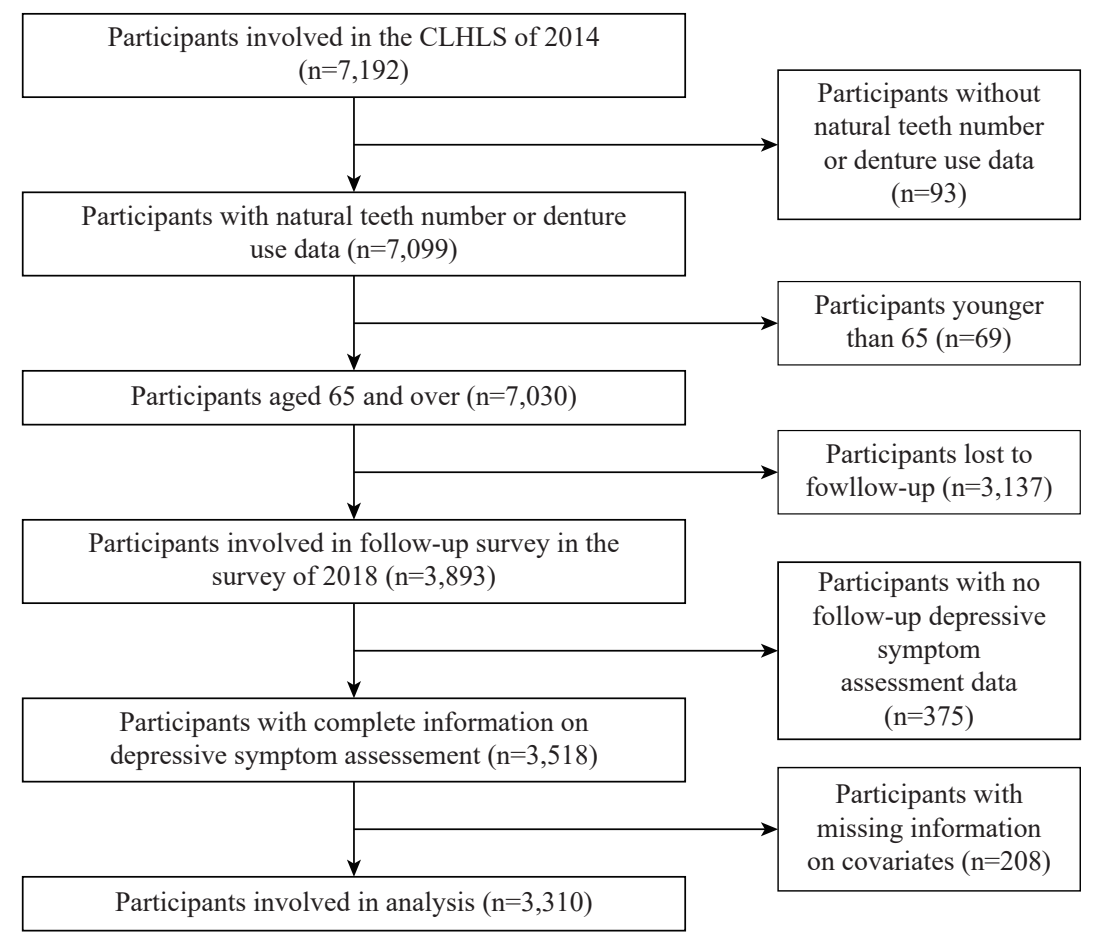

SUPPLEMENTARY FIGURE S1. Flow chart of sample selection.

Abbreviation: CLHLS=Chinese Longitudinal Healthy Longevity Survey. 
SUPPLEMENTARY TABLE S1. Baseline characteristics of the included participants.

\begin{tabular}{|c|c|c|c|c|c|c|}
\hline \multirow{2}{*}{ Item } & \multirow[b]{2}{*}{ Overall } & \multicolumn{5}{|c|}{ Number of natural teeth $(\mathrm{N}=3,310)$} \\
\hline & & $20+$ & $10-19$ & $0-9$ & 0 & $P$-value \\
\hline Number of participants, $\mathrm{n}(\%)$ & $3,310(100.00)$ & $846(25.56)$ & $654(19.76)$ & $870(26.28)$ & $940(28.40)$ & \\
\hline Number of natural teeth, mean (SD) & $10.56(10.30)$ & $25.69(3.94)$ & $13.71(2.89)$ & $4.68(2.69)$ & $0.00(0.00)$ & $<0.001$ \\
\hline \multicolumn{7}{|l|}{ Denture usage $(\%)$} \\
\hline Yes & $1,304(39.40)$ & $158(18.68)$ & 219(33.49) & $322(37.01)$ & $605(64.36)$ & $<0.001$ \\
\hline No & $2,006(60.60)$ & $688(81.32)$ & $435(66.51)$ & $548(62.99)$ & $335(35.64)$ & \\
\hline Depressive symptom, mean (SD) & $6.78(3.19)$ & $6.09(3.10)$ & $6.97(3.15)$ & $7.20(3.22)$ & $6.85(3.17)$ & $<0.001$ \\
\hline Age, mean (SD) & $81.29(10.32)$ & $74.21(8.10)$ & $79.10(8.90)$ & $84.54(9.45)$ & 86.23(9.90) & $<0.001$ \\
\hline Male, n (\%) & $1,576(46.99)$ & $490(57.36)$ & $323(48.04)$ & $369(44.15)$ & 394(39.33) & $<0.001$ \\
\hline Married, n (\%) & $1,639(46.92)$ & $549(68.97)$ & $367(50.87)$ & $366(36.95)$ & $357(33.57)$ & $<0.001$ \\
\hline Urban residence, n (\%) & $1,426(39.13)$ & $387(41.26)$ & $291(39.7)$ & $370(37.20)$ & $378(39.16)$ & 0.093 \\
\hline Years of education, mean (SD) & $2.53(3.65)$ & $3.87(4.02)$ & $2.66(3.75)$ & $1.88(3.27)$ & $1.86(3.18)$ & $<0.001$ \\
\hline Have pension, $\mathrm{n}(\%)$ & 1,191(35.98) & $367(43.38)$ & $254(38.84)$ & $311(35.75)$ & $259(27.55)$ & $<0.001$ \\
\hline \multicolumn{7}{|l|}{ Smoking, $\mathrm{n}(\%)$} \\
\hline Current smoker & $586(20.98)$ & 17226.78) & $112(18.82)$ & 141(19.41) & $161(18.75)$ & $<0.001$ \\
\hline Former smoker & $385(15.66)$ & $121(16.84)$ & $81(16.79)$ & $88(14.75)$ & $95(14.66)$ & \\
\hline Non-smoker & $2,319(63.36)$ & $549(56.38)$ & $459(64.39)$ & $635(65.84)$ & $676(66.59)$ & \\
\hline \multicolumn{7}{|l|}{ Drinking, $\mathrm{n}(\%)$} \\
\hline Current drinker & $558(20.43)$ & $174(24.60)$ & $111(20.86)$ & $128(18.50)$ & $145(18.20)$ & $<0.001$ \\
\hline Former drinker & $289(13.05)$ & $91(14.89)$ & $56(13.81)$ & $73(13.38)$ & $69(10.34)$ & \\
\hline Non-drinker & $2,425(66.52)$ & $571(60.52)$ & $480(65.33)$ & $656(68.12)$ & $718(71.46)$ & \\
\hline Frequent fruit intake, $\mathrm{n}(\%)$ & $487(13.12)$ & 134(16.49) & $85(10.61)$ & 119(10.39) & $149(14.77)$ & $<0.001$ \\
\hline Frequent vegetable intake, $\mathrm{n}(\%)$ & $521(22.13)$ & $162(24.50)$ & $87(25.24)$ & $129(20.27)$ & $143(19.52)$ & $<0.001$ \\
\hline Restricted ADL, n (\%) & $300(5.61)$ & $45(2.53)$ & $41(3.27)$ & $85(6.54)$ & $129(9.35)$ & $<0.001$ \\
\hline MMSE score, mean (SD) & $25.95(4.87)$ & $27.73(3.18)$ & $26.59(4.11)$ & $25.04(5.38)$ & $24.75(5.52)$ & $<0.001$ \\
\hline Playing card/mahjong, n (\%) & $276(7.84)$ & $106(10.80)$ & $52(7.41)$ & $59(6.89)$ & $59(6.36)$ & $<0.001$ \\
\hline Community activity, $\mathrm{n}(\%)$ & $125(3.38)$ & $45(4.89)$ & $26(3.49)$ & $28(2.69)$ & $26(2.60)$ & $<0.001$ \\
\hline Travel, $\mathrm{n}(\%)$ & $287(7.00)$ & $113(11.09)$ & $55(6.83)$ & $65(5.22)$ & $54(5.14)$ & $<0.001$ \\
\hline
\end{tabular}

Note: $P$ values are calculated with analysis of variance (ANOVA) for continuous variables and Chi-squared test for categorical variable. Abbreviations: $A D L=A c t i v i t i e s$ of daily living; $M M S E=M i n i-m e n t a l$ state examination.

drinking (current drinker, former drinker or non-drinker), frequent fruit intake (yes or no), frequent vegetable intake (yes or no), Activities of daily living (ADL) (restricted or not), and mini-mental state examination (MMSE) score. The third group were made up of social engagement variables, including playing cards or mahjong (yes or no), participation in community activities (yes or no), and travel (yes or no). 
SUPPLEMENTARY TABLE S2. Depressive symptom scores by covariates $(N=3,310)$.

Item

Depressive symptoms, mean (SD)

Sex

Female

$6.57(3.28)$

Male

$5.75(3.19)$

Age, years

$65-74$

$5.90(3.11)$

$75+$

$6.27(3.31)$

Ethnicity

Han

$6.12(3.20)$

Minority

$6.19(3.30)$

Marital status

Married

$5.74(3.11)$

Unmarried or widowed

$6.66(3.35)$

Pension

Yes

$5.83(3.23)$

No

6.36(3.26)

Residence

Urban

5.72(3.32)

Rural

6.51(3.17)

Denture usage

Yes

$5.95(3.27)$

No

$6.29(3.25)$

Smoking

Current smoker

5.92(3.20)

Former smoker

$5.24(3.27)$

Non-smoker

$6.39(3.24)$

Drinking

Current drinker

5.56(3.17)

Former drinker

5.54(3.32)

Non-drinker

$6.38(3.25)$

Frequent fruit intake

Yes

4.87(3.19)

No

6.39(3.22)

Frequent vegetable intake

Yes

$5.77(3.33)$

No

$6.24(3.25)$

Restricted ADL

Yes

$6.83(3.17)$

No

6.10(3.27)

Playing card/mahjong

Yes

$4.64(2.93)$

No

6.31(3.25)

Community activity

Yes

$4.89(3.10)$

No

6.22(3.26)

Travel

Yes

4.51(2.92)

No

6.34(3.25)

Abbreviation: $A D L=$ Activities of daily living. 


\section{REFERENCES}

1. Yi Z, Vaupelly JW. Association of late childbearing with healthy longevity among the oldest-old in China. Popul Stud 2004;58(1):37 - 53. http://dx.doi.org/10.1080/0032472032000175437.

2. Shen K, Zhang B, Feng QS. Association between tea consumption and depressive symptom among Chinese older adults. BMC Geriatr 2019;19(1):246. http://dx.doi.org/10.1186/s12877-019-1259-z.

3. Li J, Xu HZ, Pan W, Wu B. Association between tooth loss and cognitive decline: a 13-year longitudinal study of Chinese older adults. PLoS One 2017;12(2):e0171404. http://dx.doi.org/10.1371/journal.pone.0171404.

4. Yang HL, Li FR, Chen PL, Cheng X, Mao C, Wu XB. Tooth Loss, denture use, and cognitive impairment in Chinese older adults: a community cohort study. J Gerontol Ser A Biol Sci Med Sci 2021. http://dx. doi.org/10.1093/gerona/glab056.Yuan JQ, Lv YB, Kraus VB, Gao X, Yin ZX, Chen HS, et al. Number of natural teeth, denture use and mortality in Chinese elderly: a population-based prospective cohort study. BMC Oral Health 2020;20(1):100. http://dx.doi.org/10.1186/s12903-020-01084-9. 